\title{
Clinical characteristics and outcomes of hematologic malignancy patients with Clostridium difficile toxin immunoassay versus PCR positive test results
}

\author{
Matthew Ziegler, MD¹, Daniel Landsburg, MD $^{2}$, David Pegues, MD $^{1,3}$, Kevin Alby, PhD ${ }^{4}$, \\ Cheryl Gilmar, MS, $\mathbf{M T}^{3}$, Kristen Bink, MSN, RN ${ }^{2}$, Theresa Gorman, MSN, RN ${ }^{2}$, Amy Moore, \\ MSN, RN² ${ }^{2}$ Brittaney Bonhomme, BA ${ }^{5}$, Jacqueline Omorogbe, BS $^{5}$, Dana Tango, MPH $^{5}$, \\ Pam Tolomeo, MPH $^{5}$, and Jennifer H. Han, MD, MSCE $1,3,5$ \\ ${ }^{1}$ Division of Infectious Diseases, University of Pennsylvania, Philadelphia, PA \\ ${ }^{2}$ Division of Hematology and Oncology, University of Pennsylvania, Philadelphia, PA \\ ${ }^{3}$ Department of Healthcare Epidemiology, Infection Prevention and Control, University of \\ Pennsylvania, Philadelphia, PA \\ ${ }^{4}$ Department of Pathology and Laboratory Medicine, University of Pennsylvania, Philadelphia, PA \\ ${ }^{5}$ Center for Clinical Epidemiology and Biostatistics, Perelman School of Medicine, University of \\ Pennsylvania, Philadelphia, PA
}

\begin{abstract}
In a cohort of inpatients with hematologic malignancy and positive enzyme immunoassay (EIA) or PCR Clostridium difficile tests, we found that clinical characteristics and outcomes were similar between both groups. The method of testing is unlikely to predict infection in this population, and PCR-positive results should be treated with concern.
\end{abstract}

\section{INTRODUCTION}

Both infection and colonization with Clostridium difficile are common in patients with hematologic malignancy, with $10-29 \%$ of patients positive by culture on admission. ${ }^{1-2}$ However, while there is increasing recognition that molecular-based testing (PCR) for $C$. difficile toxin lacks specificity for detecting infection as opposed to colonization, 3,4 determining true infection in patients with hematologic malignancy may be particularly difficult given the high prevalence of diarrhea due to other etiologies (e.g., chemotherapy, antibiotics $)^{5,6}$ and absence of typical signs and symptoms of infection such as leukocytosis or fever due to the effect of disease and/or therapy. Similarly, while studies have suggested lower rates of both characteristics predictive of infection and poor outcomes in patients with PCR versus enzyme immunoassay (EIA) positive tests, ${ }^{7,8}$ it is unknown if these findings apply to patients with hematologic malignancy. Therefore, we aimed to compare clinical

Corresponding Author: Matthew Ziegler, MD, Address: 3400 Spruce St. 3 Silverstein, Suite E Philadelphia, PA 19104, Phone: (215) 615-1717, Fax: (215) 662-7611, matthew.ziegler@uphs.upenn.edu.

Potential conflicts of interest: All authors report no conflicts of interest relevant to this study. 
characteristics and outcomes between patients with EIA versus PCR positive $C$. difficile test results in a cohort of inpatients with hematologic malignancy.

\section{METHODS}

We performed a retrospective cohort study of patients admitted to the Hospital of the University of Pennsylvania (HUP), a 776-bed tertiary care medical center from January 1, 2015 to March 31, 2017. Patients with active hematologic malignancy and a positive C.difficile test during hospitalization were included.

Stool samples ordered for $C$. difficile testing were processed by the HUP Clinical Microbiology Laboratory. The testing algorithm uses a commercial EIA for detection of toxin A, B, and glutamate dehydrogenase (GDH) (C Diff Quik Check Complete ${ }^{\mathrm{TM}}$, Alere). Samples which are negative for toxin $\mathrm{A}$ and $\mathrm{B}$, but positive for GDH are subsequently tested using PCR for toxin genes (BD MAX ${ }^{\mathrm{TM}}$ Cdiff Assay, Becton Dickinson).

Clinical data were collected using medical record review, including demographics, comorbidities, antibiotic use in the previous month, clinical signs and symptoms (including fever, diarrhea, number of bowel movements, abdominal pain, and imaging evidence of colitis) and medication use in the 72 hours prior to the positive test. Clinical outcomes were also collected, including toxic megacolon, colectomy, recurrent $C$.difficile disease in the 90 days after index testing, as well as all-cause intensive care unit (ICU) transfer, in-hospital mortality, and hospital readmission. Clinical characteristics and outcomes of patients with EIA versus PCR positive $C$. difficile test results were compared using chi-square or Fischer's exact test for categorical variables and the Wilcoxon rank-sum test for continuous variables (Stata 14.2, StataCorp LC, College Station, TX). For all calculations, a 2-tailed $P$ value $<0.05$ was considered to be significant.

\section{RESULTS}

Over the 27-month study period in the hospital's dedicated hematology oncology units, $11.6 \%$ of $C$.difficile tests were positive. Of the 182 patients admitted with hematologic malignancy who had a positive $C$.difficile test result, $101(55 \%)$ patients had a PCR (+)/EIA $(-)$ result, and $81(45 \%)$ had an EIA (+) result. Among patients without neutropenia, leukocytosis (white blood cell count $>15$ thousand cells $/ \mathrm{mm}^{3}$ ) at the time of testing was significantly more common in the EIA (+) group (26\%) versus PCR (+)/EIA (-) group $(11 \%)(P=0.02)$ (Table 1). There was no difference in rates of severe $\mathrm{CDI}^{9}$, fever, diarrhea, or imaging evidence of colitis between the two groups. Stool output trended towards being higher in the PCR (+)/EIA (-) group, with a median of 4 bowel movements per 24 hours compared to a median of 3 bowel movements per 24 hours in the EIA $(+)$ group $(P=0.15)$.

Receipt of medications associated with an increased risk for $C$.difficile infection, including acid suppressants (52\%) and systemic antibiotics (80\%), were similar in both groups. There were relatively high rates of recent use of laxatives (30\%), but this was not significantly different between the two groups. 
We observed high rates of adverse outcomes in the cohort, including an in-hospital mortality rate of $18 \%$ and ICU transfer rate of $25 \%$, but these were similar between the two groups (Table 2). Toxic megacolon was uncommon, but occurred in $2(2 \%)$ of patients in the PCR $(+) /$ EIA $(-)$ group compared to $0(0 \%)$ in the EIA $(+)$ group $(P=0.20)$. Most patients received treatment with oral vancomycin (59\%). Two patients in the PCR (+)/EIA (-) group did not receive treatment; neither developed a measured adverse outcome.

\section{DISCUSSION}

We compared clinical characteristics and outcomes in patients with hematologic malignancy and an EIA versus PCR positive $C$. difficile test result after positive GDH screening. We demonstrate that clinical characteristics and outcomes are similar in this cohort, whether results are positive by EIA or PCR. In addition, the results of our study highlight the significant morbidity and mortality of patients with $C$. difficile in this population, with high rates of ICU transfer and death.

Particularly in a population characterized by high rates of colonization with $C$. difficile, ${ }^{1,2}$ it is important to differentiate infection versus colonization. However, our results suggest that among patients with hematologic malignancy, the testing modality (i.e., EIA versus PCR) cannot be used to reliably distinguish between $C$. difficile infection or colonization. Specifically, clinical factors typically associated with active or more severe infection ${ }^{9}$ were similar between the two groups. Complicating the appropriate diagnosis of $C$. difficile infection in this population, there was a high rate of use of laxative and stool softeners in the 72 hours prior to $C$. difficile testing in both groups.

Clinical outcomes were also similar between hematologic malignancy patients with PCR $(+) /$ EIA (-) versus EIA(+) C. difficile test results. Morbidity and mortality were high, likely reflecting the overall complexity and severity of illness of patients hospitalized with hematologic malignancy. However, those outcomes specific to CDI were also similar between both groups, with rates of recurrent $C$.difficile infection of $12 \%$ within 90 days, and cases of toxic megacolon identified in the PCR (+)/EIA (-) group.

Our results differ from studies of general medical patients that have found those with toxin EIA(+) $C$. difficile results to have both a greater prevalence of CDI clinical characteristics and worse outcomes compared to PCR (+)/EIA (-) results ${ }^{7,8}$. A prospective study without GDH screening found those with PCR (+)/EIA (-) results to have a lower prevalence of leukocytosis, fewer number of stools, and lower rates of adverse outcomes, including mortality and recurrent $\mathrm{CDI}^{7}$. However, the 30 -day mortality of $0.6 \%$ in the PCR (+)/EIA $(-)$ group in this study compared to $15 \%$ in our study highlights the significant difference in study populations. Another recent study also demonstrated higher rates of leukocytosis, fever, and severe CDI as well as recurrent $C$.difficile infection with an EIA(+) result versus PCR (+)/EIA (-) result after GDH screening, but did not find a difference in mortality between the groups ${ }^{8}$. Notably, our study included only samples collected through routine clinical care and were tested via a multistage process which included a C.difficile GDH screening test. While we are comparing EIA and PCR test results, these are among patients who have had a positive GDH screen. In a multi-center study comparing clinical outcomes 
among general medical patients, GDH screening was shown to perform similarly to cytotoxigenic culture and had similar sensitivity to $\mathrm{PCR}^{10}$. However, it is possible that our results differ somewhat from prior studies where GDH screening was not performed.

Our study has potential limitations. First, given the relatively limited sample size available for clinical outcomes, we were unable to perform multivariable analysis for the association between $C$. difficile testing method and patient outcomes. Additionally, our study focused on the care of hematology oncology patients at an academic institution and may not be generalizable to populations with different characteristics.

In conclusion, our findings highlight the importance of evaluating the characteristics and performance of $C$. difficile testing algorithms specifically in high-risk populations. Additionally, considering the high morbidity and mortality associated with $C$. difficile in this population, future studies are needed focusing on optimal methods of differentiating colonization versus infection, as well as preventing $C$. difficile disease in patients with hematologic malignancy ${ }^{11}$.

\section{Acknowledgments}

Financial support: This work was supported by the National Institutes of Health (grant no. T32-AI055435 to M.Z. and grant no. K01-AI103028 to J.H.H.) and by a CDC Cooperative Agreement, FOA\#CK16-004-Epicenters for the Prevention of Healthcare Associated Infections. The funding agencies had no role in the design and conduct of the study; collection, management, analysis, and interpretation of the data; or preparation, review, or approval of the manuscript.

The interim results of this study were presented at the Infectious Disease Society of America IDWeek as a poster (Presentation \#1291) on October 6th, in San Diego, California

\section{References}

1. Bruminhent J, Wang ZX, Hu C, et al. Clostridium difficile colonization and disease in patients undergoing hematopoietic stem cell transplantation. Biol Blood Marrow Transplant. 2014; 20:1329_ 1334. [PubMed: 24792871]

2. Jain T, Croswell C, Urday-Cornejo V, et al. Clostridium difficile colonization in hematopoietic stem cell transplant recipients: A prospective study of the epidemiology and outcomes involving toxigenic and nontoxigenic strains. Biol Blood Marrow Transplant. 2016; 22:157-163. [PubMed: 26211988]

3. Dubberke ER, Han Z, Bobo L, et al. Impact of clinical symptoms on interpretation of diagnostic assays for Clostridium difficile infections. J Clin Microbiol. 2011; 49:2887-2893. [PubMed: 21697328]

4. Truong C, Schroeder LF, Gaur R, et al. Clostridium difficile rates in asymptomatic and symptomatic hospitalized patients using nucleic acid testing. Diagn Microbiol Infect Dis. 2017; 87:365-370. [PubMed: 28087170]

5. Akahoshi Y, Kimura S, Nakano H, et al. Significance of a positive Clostridium difficile toxin test after hematopoietic stem cell transplantation. Clin Transplant. 2016; 30:703-708. [PubMed: 27019071]

6. Stein A, Voigt W, Jordan K. Chemotherapy-induced diarrhea: pathophysiology, frequency and guideline-based management. Ther Adv Med Oncol. 2010; 2:51-63. [PubMed: 21789126]

7. Polage CR, Gyorke CE, Kennedy MA, et al. Overdiagnosis of clostridium difficile infection in the molecular test era. JAMA Intern Med. 2015; 175:1792-1801. [PubMed: 26348734]

8. Origuen J, Corbella L, Orellana MA, et al. Comparison of the clinical course of Clostridium difficile infection in gdh-positive, toxin-negative patients diagnosed by PCR to those with a positive toxin test. Clin Microbiol Infect. (in press). 
9. Surawicz CM, Brandt LJ, Binion DG, et al. Guidelines for diagnosis, treatment, and prevention of clostridium difficile infections. Am J Gastroenterol. 2013; 108:478-498. quiz 499. [PubMed: 23439232]

10. Planche TD, Davies KA, Coen PG, et al. Differences in outcome according to Clostridium difficile testing method: a prospective multicentre diagnostic validation study of C difficile infection.

Lancet Infect Dis. 2013; 13(11):936-945. [PubMed: 24007915]

11. Chakrabarti S, Lees A, Jones SG, Milligan DW. Clostridium difficile infection in allogeneic stem cell transplant recipients is associated with severe graft-versus-host disease and non-relapse mortality. Bone Marrow Transplant. 2000; 26:871-876. [PubMed: 11081387] 


\section{TABLE 1}

Clinical Characteristics of patients with hematologic malignancy with EIA versus PCR positive C.difficile test results

\begin{tabular}{|c|c|c|c|c|}
\hline Characteristics & $\begin{array}{l}\text { Total Population } \\
\text { No. }(\%) \\
\text { n=182 }\end{array}$ & $\begin{array}{c}\text { EIA Positive } \\
\text { No. }(\%) \\
\text { n=81 }\end{array}$ & $\begin{array}{c}\text { PCR Positive } \\
\text { No. }(\%) \\
\text { n=101 }\end{array}$ & $\underset{\text { Value }}{P}$ \\
\hline Age & $62(53-68)^{a}$ & $62(55-68)^{a}$ & $62(52-68)^{a}$ & 0.74 \\
\hline White race & $140(77)$ & $61(75)$ & $79(78)$ & 0.64 \\
\hline \multicolumn{5}{|l|}{ Malignancy } \\
\hline Acute myeloid leukemia & $81(45)$ & $36(44)$ & $45(45)$ & \multirow{4}{*}{0.91} \\
\hline Multiple myeloma & $41(23)$ & $19(23)$ & $22(22)$ & \\
\hline Non-Hodgkin's lymphoma & $26(14)$ & $10(12)$ & $16(16)$ & \\
\hline Other & $34(19)$ & $16(20)$ & $18(18)$ & \\
\hline C.difficile test collected $<72 \mathrm{hrs}$ within admission & $33(18)$ & $17(21)$ & $16(16)$ & 0.37 \\
\hline History of stem cell transplant & $67(37)$ & $35(43)$ & $32(32)$ & 0.11 \\
\hline History of $C$.difficile $b$ & $22(12)$ & $13(16)$ & $9(9)$ & 0.14 \\
\hline Prior hospitalization $^{c}$ & $103(57)$ & $48(59)$ & $55(54)$ & 0.51 \\
\hline Chronic gastrointestinal disease ${ }^{d}$ & $32(18)$ & $18(23)$ & $14(14)$ & 0.13 \\
\hline Neutropenia $e$ & $64(35)$ & $24(30)$ & $40(40)$ & 0.14 \\
\hline Leukocytosis $f$ & $21(18)$ & $15(26)$ & $6(11)$ & 0.02 \\
\hline Fever ${ }^{g}$ & $68(37)$ & $27(33)$ & $41(41)$ & 0.31 \\
\hline Albumin $h$ & $2.9(2.4-3.4)$ & $2.7(2.2-3.3)$ & $3.0(2.5-3.5)$ & 0.11 \\
\hline Severe $C$.difficile infection ${ }^{i}$ & $22(12)$ & $13(16)$ & $9(9)$ & 0.14 \\
\hline Diarrhea $j$ & $133(73)$ & $57(70)$ & $76(75)$ & 0.46 \\
\hline Stool count ${ }^{k}$ & $3(2-5)^{a}$ & $3(2-5)^{a}$ & $4(2-6)^{a}$ & 0.15 \\
\hline Radiographic evidence of colitis & $15(8)$ & $6(7)$ & $9(9)$ & 0.71 \\
\hline \multicolumn{5}{|l|}{ Medications ${ }^{l}$} \\
\hline Proton-pump inhibitor & $73(40)$ & $32(40)$ & $41(41)$ & 0.93 \\
\hline Histamine-2 antagonist & $30(16)$ & $15(19)$ & $15(15)$ & 0.50 \\
\hline Corticosteroid & $73(40)$ & $39(48)$ & $34(34)$ & 0.05 \\
\hline Loperamide & $10(6)$ & $5(6)$ & $5(5)$ & 0.74 \\
\hline
\end{tabular}

Infect Control Hosp Epidemiol. Author manuscript; available in PMC 2019 July 01. 


\begin{tabular}{lcccc}
\hline Characteristics & $\begin{array}{c}\text { Total Population } \\
\text { No. (\%) } \\
\mathbf{n = 1 8 2}\end{array}$ & $\begin{array}{c}\text { EIA Positive } \\
\text { No. (\%) } \\
\mathbf{n = 8 1}\end{array}$ & $\begin{array}{c}\text { PCR Positive } \\
\text { No. (\%) } \\
\mathbf{n = 1 0 1}\end{array}$ & $\begin{array}{c}\boldsymbol{P} \\
\text { Value }\end{array}$ \\
\hline Laxative $^{m}$ & $54(30)$ & $21(26)$ & $33(33)$ & 0.32 \\
\hline Docusate & $46(25)$ & $19(23)$ & $27(27)$ & 0.61 \\
\hline Antibiotics & $\mathcal{1}$ & & & \\
\hline Any antibiotic & $145(80)$ & $65(80)$ & $80(79)$ & 0.83 \\
\hline $\begin{array}{c}\text { Anti-pseudomonal } \\
\text { Antibiotic }\end{array}$ & $119(65)$ & $55(68)$ & $64(64)$ & 0.52 \\
\hline
\end{tabular}

NOTE. EIA, enzyme immunoassay; PCR, polymerase chain reaction

${ }^{a}$ Median, inter-quartile range (IQR)

${ }^{b}$ A positive C.difficile test by PCR or EIA within the prior year

${ }^{c}$ Within the prior 30 days

${ }^{d}$ Graft-versus-host disease, inflammatory bowel disease (Crohn's disease or ulcerative colitis), irritable bowel syndrome, short gut syndrome

${ }^{e}$ Absolute neutrophil count $<500$ cells $/ \mathrm{mm}^{3}$ within 72 hours of the index $C$. difficile test

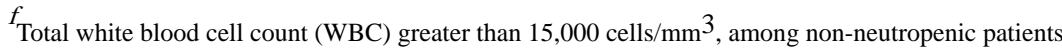

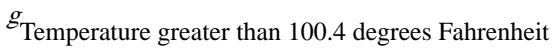

$h_{\text {Within }} 72$ hours, $\mathrm{n}=110$

${ }^{i}$ Serum albumin $\left\langle 3 \mathrm{~g} / \mathrm{dl}\right.$ plus $\mathrm{WBC}>=15,000$ cells $/ \mathrm{mm}^{3}$ or abdominal tenderness

$j_{\text {Listed as diarrhea or liquid stool by provider }}$

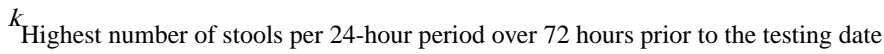

${ }^{I}$ Within the previous 72 hours of the testing date

$m_{\text {Includes sennosides, polyethylene glycol, milk of magnesia, bisacodyl, lactulose }}$

${ }^{n}$ Cefepime, meropenem, piperacillin-tazobactam, and levofloxacin 
TABLE 2

Outcomes of patients with hematologic malignancy and EIA versus PCR positive C.difficile test results

\begin{tabular}{|c|c|c|c|c|}
\hline Outcomes & $\begin{array}{c}\text { Total Population } \\
\text { No. }(\%) \\
\text { n=182 }\end{array}$ & $\begin{array}{c}\text { EIA Positive } \\
\text { No. }(\%) \\
\mathbf{n}=\mathbf{8 1}\end{array}$ & $\begin{array}{c}\text { PCR Positive } \\
\text { No. }(\%) \\
\text { n=101 }\end{array}$ & $P$ Value \\
\hline In-hospital mortality ${ }^{a}$ & $33(18)$ & $18(23)$ & $15(15)$ & 0.18 \\
\hline ICU transfer $b$ & $45(25)$ & $23(28)$ & $22(22)$ & 0.30 \\
\hline Toxic megacolon & $2(1)$ & $0(0)$ & $2(2)$ & 0.20 \\
\hline Colectomy & $4(2)$ & $1(1)$ & $3(3)$ & 0.42 \\
\hline C.difficile recurrence & $21(12)$ & $7(9)$ & $14(14)$ & 0.27 \\
\hline GVHD of the GI tract & $11(6)$ & $6(7)$ & $5(5)$ & 0.48 \\
\hline \multicolumn{5}{|l|}{ Treatment $c$} \\
\hline None & $4(2)$ & $0(0)$ & $4(4)$ & 0.07 \\
\hline Oral vancomycin & $118(65)$ & $57(70)$ & $61(60)$ & 0.16 \\
\hline Days & $15(10-21)^{d}$ & $15(10-22)^{d}$ & $14(10-21)^{d}$ & 0.83 \\
\hline $\begin{array}{l}\text { Oral } \\
\text { metronidazole }\end{array}$ & $107(59)$ & $43(53)$ & $64(63)$ & 0.16 \\
\hline Days & $10(4-14)^{d}$ & $8(3-14)^{d}$ & $11(6-15)^{d}$ & 0.03 \\
\hline $\begin{array}{r}\text { Intravenous } \\
\text { metronidazole }\end{array}$ & $49(27)$ & $21(26)$ & $28(28)$ & 0.79 \\
\hline Days & $6(3-12)^{d}$ & $6.5(3.5-9.5)^{d}$ & $6(3-15)^{d}$ & 0.64 \\
\hline
\end{tabular}

NOTE. EIA, enzyme immunoassay; PCR, polymerase chain reaction; ICU, intensive care unit; GVHD, graft-versus-host disease; GI, gastrointestinal

Within 90 days

$b_{\text {Within } 30 \text { days }}$

$c$ Patients may have received more than one antibiotic for treatment

${ }^{d}$ Median, inter-quartile range (IQR) 